\title{
OPEN Mobile sonouroflowmetry using voiding sound and volume
}

\author{
Elie El Helou ${ }^{1 \bowtie}$, Joy Naba ${ }^{1}$, Karim Youssef ${ }^{2}$, Georges Mjaess ${ }^{1}$, Ghassan Sleilaty ${ }^{1}$ \& \\ Samar Helou ${ }^{3 \otimes}$
}

Uroflowmetry (UF) is a common clinic-based non-invasive test to diagnose Lower Urinary Tract Dysfunction (LUTD). Accurate home-based uroflowmetry methods are needed to conveniently conduct repeated uroflowmetries when patients are physiologically ready to urinate. To this end, we propose and evaluate a novel mobile sonouroflowmetry (SUF) method that estimates the urinary flow rate from a sound signal recorded using a mobile phone. By linearly mapping the total sound energy to the total voided volume, the sound energy curve is transformed to a flow rate curve allowing the estimation of the flow rate over time. An evaluation using data from 44 healthy young men showed high similarity between the UF and SUF flow rates with a mixed-effects model correlation coefficient of 0.993 and a mean root mean square error of $2.37 \mathrm{ml} / \mathrm{s}$. Maximum flow rates were estimated with an average absolute error of $2.41 \mathrm{ml} / \mathrm{s}$. Future work on mobile uroflowmetry can use these results as an initial benchmark for flow rate estimation accuracy.

Lower Urinary Tract Dysfunction (LUTD) is a common complaint that can impact a patient's quality of life and cause a substantial economic burden ${ }^{1}$. LUTD can be the result of numerous conditions including bladder outlet obstruction and over- or under-active bladder-which in turn can be caused by various conditions including benign prostatic hyperplasia and urethral stricture, neurologic diseases, diabetes, and pharmacologic treatment ${ }^{2}$. In recent decades, functional studies of the bladder and its outlet have become more widespread, rendering the assessment of LUTD more objective ${ }^{3}$. Among these diagnostic modalities, uroflowmetry (UF) is the easiest non-invasive LUTD assessment tool ${ }^{4,5}$, and is often considered sufficient for diagnosis and treatment of LUTD, along with history-taking and clinical examination ${ }^{6}$.

The important elements of a uroflowmetry test are the total voided volume $\left(\mathrm{V}_{\mathrm{t}}\right)$ (which should be $>150 \mathrm{ml}$ ), the maximum flow rate $\left(\mathrm{Q}_{\max }\right)$, and the curve of the flow rate (which should be bell shaped $)^{7}$. Conducting a uroflowmetry involves the patient urinating in a flowmeter at a predetermined time. This process is unnatural and requires "on-demand" urination, often with low or very high bladder filling, instead of urinating when physiologically ready. This results in non-reproducible flow measurements ${ }^{8,9}$, hence the difficulty of diagnosis and therapeutic adaptation ${ }^{10}$. To get reproducible measurements of $Q_{\max }, Q_{\max }$ can be adjusted to $V_{t}$ as a hyperbolic relationship exists between the two parameters ${ }^{8}$. However, the relationship between $Q_{\max }$ and $V_{t}$ differs substantially between patients ${ }^{8}$. Therefore, to get accurate flow measurements, it has been recommended to repeat uroflowmetry tests for each patient, which requires multiple time-consuming and costly clinical visits ${ }^{8,11-13}$.

To address the limitations of conventional uroflowmetry, home-based uroflowmetry methods were proposed. To have reliable home measurements of $\mathrm{Q}_{\max }$, it was estimated that as much as 25 flows are needed ${ }^{14}$. However, there are currently no cheap, practical, and accurate home-based methods that estimate the flow rate over time ${ }^{15-17}$. Promising approaches for home-based uroflowmetry are sound-based and vision-based ${ }^{17}$. A recent study showed that computer vision can be used to estimate the urinary flow rate. However, this requires the installation of a high-speed camera ${ }^{18}$. In comparison, mobile sonouroflowmetry (SUF) methods would be more practical, as they allow the extraction of urinary flow parameters from a mobile phone recording of the sound generated by the urine stream hitting a surface ${ }^{10}$. Even though it has long been recognized that the sound of a patient urinating can be used to diagnose LUTD ${ }^{19-22}$, sonouroflowmetry remains seldom studied in the literature.

Early experimental work validated the concept of mobile sonouroflowmetry by showing that the acoustic signatures of the sound generated by the urine stream are correlated with the urinary flow rate ${ }^{23,24}$; however, few studies have explored its feasibility and validity since 2011 . Recent studies ${ }^{10,25}$ revalidated the basic concept and described high correlations between the intensity of the sound signal and the flow parameters measured using a uroflowmetry device. In 2021, Lee et al. ${ }^{26}$ evaluated the reliability of a smartphone-based sonouroflowmetry method in a population of male and female subjects with and without LUTD. The authors reported strong

${ }^{1}$ Faculty of Medicine, Saint Joseph University, Beirut, Lebanon. ${ }^{2}$ College of Engineering and Technology, American University of the Middle East, Egaila, Kuwait. ${ }^{3} \mathrm{Global}$ Center for Medical Engineering and Informatics, Osaka University, Osaka, Japan. ${ }^{\boxplus}$ email: elie.helou@usj.edu.lb; samar@bpe.es.osaka-u.ac.jp 
correlations between $\mathrm{Q}_{\max }, \mathrm{Q}_{\mathrm{avg}}$, and $\mathrm{V}_{\mathrm{t}}$, and a strong visual correlation between flow curve patterns obtained with their SUF method and those obtained with a conventional UF device. However, the methods used to estimate the flow parameters were not fully described. In summary, existing results imply the existence of associations between UF and SUF flow parameters ${ }^{10,26}$ but do not detail the accuracy of the estimation methods. Moreover, the existing literature does not currently describe any reproducible SUF methods that allow the estimation of flow elements.

The aim of this study is to propose and evaluate the accuracy of a mobile sonouroflowmetry method. As inputs, the method takes the total voided volume $\left(\mathrm{V}_{\mathrm{t}}\right)$ and the mobile phone recording of the sound generated by the urine stream hitting water. The sound energy is assumed to be proportional to the flow rate at each moment, which implies that the total amount of sound energy is proportional to the total voided volume. Based on this assumption, the flow rate curve is generated and the $\mathrm{Q}_{\max }$ is estimated. Finally, the accuracy of the sonouroflowmetry method is systematically examined and reported. In short, the contributions of this work are:

- A sonouroflowmetry method to estimate the flow rate from a sound signal recorded using a mobile phone.

- A systematic evaluation approach and an initial benchmark for mobile uroflowmetry methods.

\section{Methods}

This study received approval from the institutional review board of Saint Joseph University, Beirut, Lebanon. The study was performed in accordance with the Declaration of Helsinki and written informed consent was obtained from all participants.

Collection of real flow parameters and sound recordings. Volunteers were recruited for this study from Hotel Dieu de France, a large university hospital in Beirut, Lebanon, between December 2019 and November 2020. Those volunteers were: (1) male subjects aged between 18 and 65 years old; (2) with no lower urinary tract symptoms and no past history of urological or neurological comorbidities; (3) having autonomous voiding habits and (4) able to urinate in a standing position.

The recruited subjects underwent a standard uroflowmetry test using a uroflowmeter from Medtronic Medical Supplies (MMS), Dublin, Ireland. The uroflowmeter uses a high accuracy digital weight sensor and transmits the data to the computer via Bluetooth with a sampling frequency of $10 \mathrm{~Hz}$. The uroflowmetry was combined with a synchronous sound recording using the native Voice Memos application on an Apple iPhone Xs Max (R), which was placed $100 \mathrm{~cm}$ away from the uroflowmetry machine. Each subject was asked to urinate, in a quiet room behind a curtain, inside a cup that was placed on the uroflowmeter and filled with $100 \mathrm{ml}$ of tap water. The used cup was the original 1-1 standardized plastic cup provided by the manufacturer-MMS-along with the uroflowmeter. The bottom diameter, top diameter, and height of the cup are 9.5, 12, and $16.5 \mathrm{~cm}$, respectively. The subjects were asked to aim on the water inside the cup-not on its borders-while urinating, in order to produce a significant sound effect. The uroflowmeter, with the cup on top, were placed on the toilet lid to create a scenario that is easy to reproduce in home settings.

For each subject, the following variables were collected:

- Demographic parameters (age and height).

- Average and maximal urine flow rate $\left(\mathrm{Q}_{\mathrm{avg}}\right.$ and $\left.\mathrm{Q}_{\max }\right)$, total voided volume $\left(\mathrm{V}_{\mathrm{t}}\right)$, voiding duration, and the flow rate curve from the uroflowmetry study.

- Mobile phone recording of the urination sound.

Sound signal processing. Cropping and filtering. The recorded sounds of the urination were made with a sampling frequency $f_{s}$ of $48 \mathrm{kHz}$. The recording was initiated before the subject started urinating and was stopped after the subject finished. Therefore, the recordings were manually cropped to remove parts not corresponding to urination. When cropping the recordings, we took into consideration the variations in the sound signal, independently from the flowrate captured by the uroflowmeter. Each recording was used as an audio signal and processed as a sequence of samples. Background noise existed in the recordings; its low-frequency components were reduced using a third-order Butterworth high-pass filter with a cutoff frequency $f_{c}$ of $1.2 \mathrm{kHz}$. The obtained signals were used in the next processing stages.

Splitting into frames. Let $s$ be one of the sound signals. $s$ is decomposed into a sequence of consecutive time frames of equal durations. As each second corresponds to $f_{s}$ samples, a frame of duration $f_{d}$ seconds comprises of $n b s=f_{d} \times f_{s}$ samples and a recording with a duration of $d$ seconds contains $n b f=\left\lfloor\frac{d}{f_{d}}\right\rfloor$ frames.

Calculating the sound energy. The measure $\mathrm{E}=\sum_{i=1}^{n b s}|f(i)|$ is calculated for each frame, where $f(i)$ is the value of the frame's $i$ th sample and |.| denotes the absolute value operator. Calculating E for all the frames of the signal $s$ leads to $n b f$ values of $E$ reflecting the variations of the sound energy in the signal. Therefore, a signal of $n b f$ frames will be represented by a sequence of $n b f$ energy values, calculated according to the previous method.

Estimation of flow parameters. Figure 1 shows four flow rate curves and their corresponding soundbased curves showing $\mathrm{E}$ in function of time. The left-hand side graph shows the flow rate curve, and the righthand side shows the E curve. The y-scales in the graphs have different units and were scaled to visually highlight the similarity between the shapes of the curves. As seen in Fig. 1, E values change in conjunction with the flow rate. Therefore, we assume that the sound energy can be used for the estimation of the flow rate. 
Flow rate curves
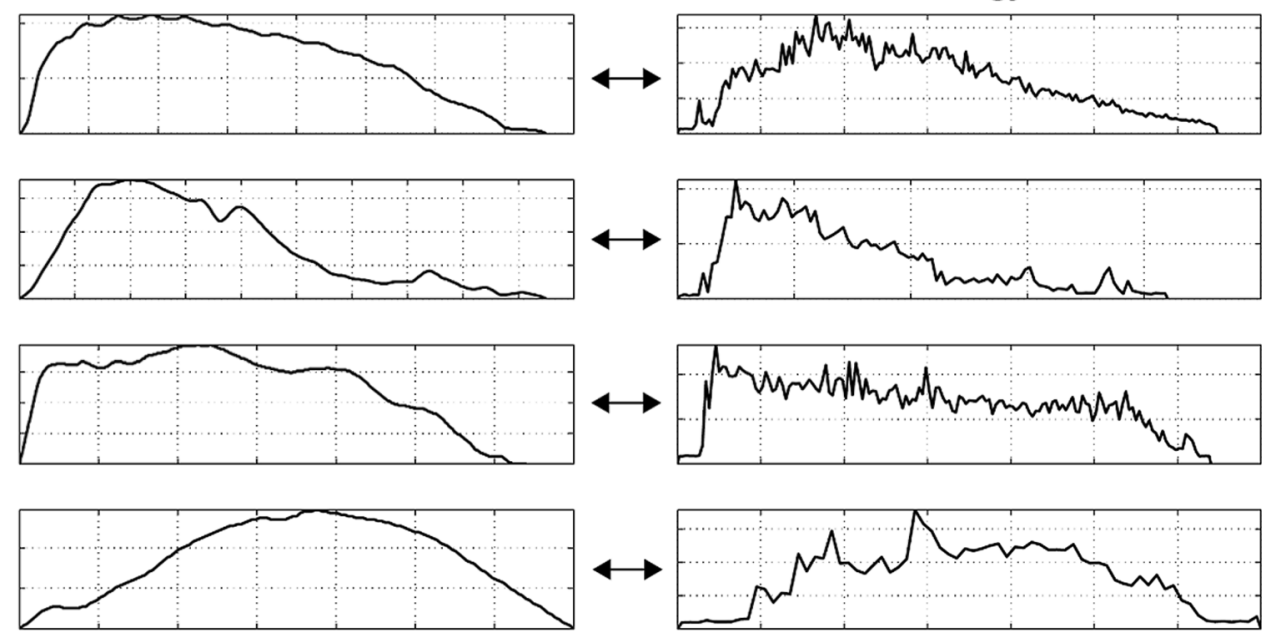

Figure 1. Flow rate and corresponding sound energy curves. The left-hand side graph shows the flow rate across time, and the right-hand side shows the corresponding sound energy across time. The $y$-scales in the graphs have different units and were scaled to visually highlight the similarity between the shapes of the curves.

As observed, $E$ varies in conjunction with the flow rate. Therefore, the area $A_{t}$ under the $E$-time curve (AUC ${ }_{\mathrm{E}}$ ) varies in conjunction with the area under the flow rate-time curve ( $\mathrm{AUC}_{\text {Flowrate }}$ ) which is equivalent to the total voided volume $V_{t}$. Normalizing the $E$-time curve by multiplying all the $E$ values by the ratio $\frac{V_{t}}{A_{t}}$ rescales this curve and makes it a sound-based representation of the flow rate curve. Accordingly, the formula used to estimate the flow rate $(Q)$ from the sound energy $(E)$ is:

$$
Q=E \times \frac{V_{t}}{A_{t}} .
$$

Once the flow rate-time curve is obtained, $\mathrm{Q}_{\max }$ can be calculated as the peak of the curve.

Figure 2 visually depicts the process of splitting the sound signal into frames, calculating the sound energy for each frame, and mapping the energy to the flow rate.

The area $A_{t}$ under the sound-based $E$-time curve is calculated as the sum of consecutive surfaces comprising it. These surfaces are triangular at both ends of the curve, and trapezoidal in between. The trapezoidal surfaces have the same width which is equal to the frame duration $f_{d}$ and heights equal to the corresponding consecutive $E$ values. This can be seen in Fig. 2 (2) and (3) showing the sequence of $E$ values as they make consecutive surface elements.

The calculated flow rates are highly dependent on the chosen frame duration. The effect of using different frame durations can be seen in Fig. S7 in the Online Appendix. Therefore, the optimal frame duration for estimating the overall flow rate curve and $\mathrm{Q}_{\max }$ may differ. Accordingly, our experiments investigate the frame durations that result in high accuracy for the overall flow rate curve and $\mathrm{Q}_{\max }$, separately.

\section{Results}

Collected dataset. Fifty-five subjects were recruited in the study. Recordings showing interfering sounds (speech, breathing, movements) were excluded. Finally, 44 subjects with a median age of 24 years old were included. Demographic and descriptive parameters of the population are presented in Table 1. Means and standard deviations (SD) were used for $\mathrm{Q}_{\max }, \mathrm{Q}_{\mathrm{avg}}$ and height (normal distribution based on Shapiro-Wilk and inspection of the Q-Q plots). Medians and interquartile range (IQR) were used for duration of voiding, voided volume, and age (non-normal distribution based on Shapiro-Wilk and inspection of the Q-Q plots).

Accuracy of flow rate estimation over time. The benchmarks for the performance of urodynamic equipment recommend a measurement bandwidth of 1 to $5 \mathrm{~Hz}$, i.e., one measurement per 0.2 to $1 \mathrm{~s}$ for uroflowmetry devices ${ }^{27}$. Accordingly, we evaluate our method for five frame durations ranging from 0.2 to $1 \mathrm{~s}$.

To study the correlation between the SUF and UF flow rate, we consider the flow rate curve to be a series of repeated flow measures and adopt a fixed-effect linear mixed-effects model. This approach allows us to account for the non-independence of the observations and for the unequal number of within-subject observations ${ }^{28,29}$. One no-intercept mixed model was developed for each frame duration, choosing an auto-regressive 1st order (AR1) covariance structure, and a restricted estimation maximum likelihood approach. Degrees of freedom estimation was approximated using the Kenward-Roger method. For each model, Schwarz's Bayesian Information Criterion (BIC) was calculated. The results are reported in Table 2.

The results show a high level of correlation between the SUF and UF flow rate values for the five frame durations. The correlation is almost perfect for a frame duration of $0.2 \mathrm{~s}(\mathrm{rho}=0.993)$, and slightly decreases with 
(1) Filtering and splitting to frames of duration $f_{d}$

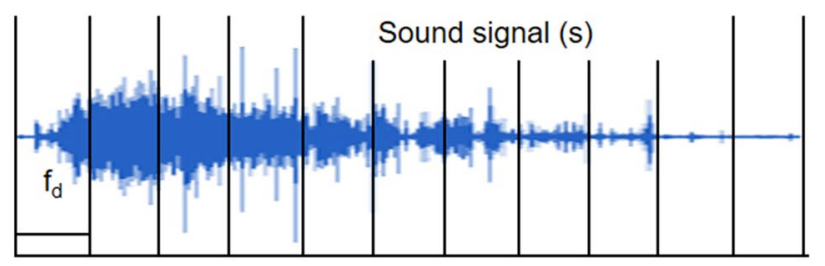

(2) Calculating the sound energy in each frame

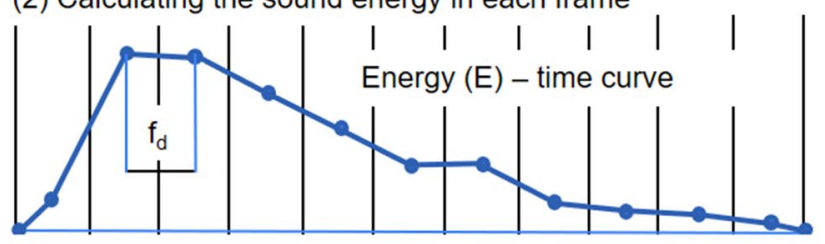

(3) Mapping the sound energy to the flow rate

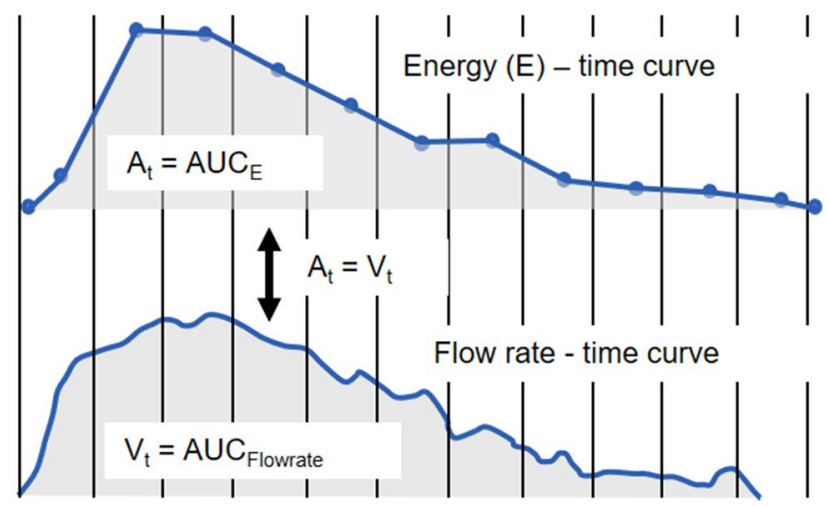

Figure 2. Transforming the sound signal to a flow rate-time curve. (1) The sound signal is split into frames of duration $\mathrm{f}_{\mathrm{d} \text { - }}(2)$ The energy is calculated for each frame and the energy-time curve is generated. This energy varies in conjunction with the flow rate and can be transformed into a flow rate curve. (3) The energy-time curve is linearly mapped to the flowrate-time curve by equating $A_{t}$, the area under the energy curve $\left(\mathrm{AUC}_{\mathrm{E}}\right)$, to the total voided volume $\mathrm{V}_{\mathrm{t}}$ which corresponds to the area under the flowrate curve $\left(\mathrm{AUC}_{\text {Flowrate }}\right)$.

\begin{tabular}{|l|c|l|l|}
\hline & Value & Minimum & Maximum \\
\hline Age, years (median $\pm \mathrm{IQR})$ & $24 \pm 1.75$ & 23 & 55 \\
\hline Height, cm (mean $\pm \mathrm{SD})$ & $178.4 \pm 7.0$ & 163 & 191 \\
\hline $\mathrm{Q}_{\max }, \mathrm{ml} / \mathrm{s}($ mean $\pm \mathrm{SD})$ & $22.4 \pm 7.7$ & 7.5 & 43.5 \\
\hline $\mathrm{Q}_{\text {avg }}, \mathrm{ml} / \mathrm{s}($ mean $\pm \mathrm{SD})$ & $12.4 \pm 4.2$ & 3.2 & 22.8 \\
\hline Voiding duration, s (median $\pm \mathrm{IQR})$ & $37.0 \pm 28.8$ & 16 & 165 \\
\hline Voided volume, $\mathrm{ml}($ median $\pm \mathrm{IQR})$ & $365 \pm 225.3$ & 82 & 947 \\
\hline
\end{tabular}

Table 1. Descriptive and demographic parameters. $S D$ standard deviation, $I Q R$ inter-quartile range.

\begin{tabular}{|l|l|l|}
\hline Frame duration & Schwarz's Bayesian criterion $($ BIC) & AR1 (rho) \\
\hline $0.2 \mathrm{~s}$ & $13,231.59$ & 0.993 \\
\hline $0.4 \mathrm{~s}$ & $10,863.08$ & 0.985 \\
\hline $0.6 \mathrm{~s}$ & 8665.11 & 0.976 \\
\hline $0.8 \mathrm{~s}$ & 7150.54 & 0.966 \\
\hline $1 \mathrm{~s}$ & 6118.56 & 0.957 \\
\hline
\end{tabular}

Table 2. Correlation between the SUF and UF flow rate. ${ }^{\star}$ Calculated from the correlation matrix for estimates of covariance parameters. 

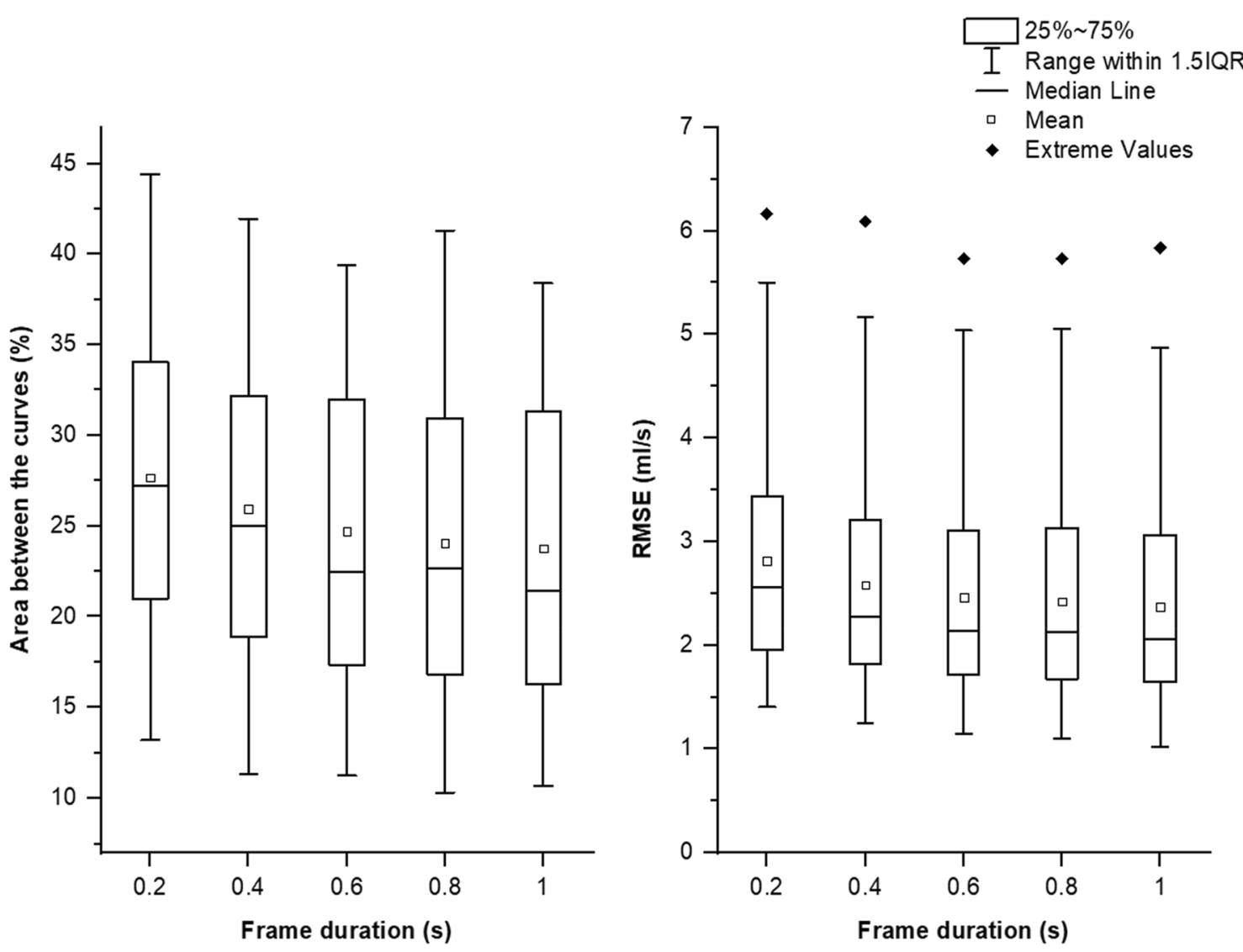

Figure 3. Comparison between the UF and SUF flow rate curves.

\begin{tabular}{|l|l|l|l|l|l|l|l|l|}
\hline \multirow{2}{*}{} & \multicolumn{6}{|l|}{ Normalized area between the curves (\%) } & \multicolumn{4}{l|}{ Root mean square error (RMSE) (ml/s) } \\
\cline { 2 - 10 } & Minimum & Maximum & Mean & SD & Minimum & Maximum & Mean & SD \\
\hline $0.2 \mathrm{~s}$ & 13.21 & 44.40 & 27.67 & 8.12 & 1.40 & 6.17 & 2.81 & 1.13 \\
\hline $0.4 \mathrm{~s}$ & 11.36 & 41.97 & 25.95 & 8.10 & 1.25 & 6.09 & 2.58 & 1.09 \\
\hline $0.6 \mathrm{~s}$ & 11.26 & 39.39 & 24.69 & 7.92 & 1.15 & 5.73 & 2.46 & 1.06 \\
\hline $0.8 \mathrm{~s}$ & 10.31 & 41.29 & 24.08 & 7.97 & 1.10 & 5.73 & 2.42 & 1.08 \\
\hline $1 \mathrm{~s}$ & 10.70 & 38.40 & 23.76 & 8.04 & 1.02 & 5.84 & 2.37 & 1.08 \\
\hline
\end{tabular}

Table 3. Comparison between the UF and SUF flow rate curves. SD standard deviation.

the increase of the frame duration. These results imply that the estimation of flow rate using the proposed SUF method is highly reliable. However, they do not imply that the UF and SUF flow rate curves have the same shape, i.e., are completely overlapping.

To assess the similarity between the shape of the UF and SUF flow rate curves, we report the area between the curves and the root mean square error (RMSE). The area between the curves was totalized and then normalized using the area under the UF flow rate curve. The results were generated by blinded processing as no pre-processing aiming to increase the alignment of the UF and SUF curves was conducted. Figure 3 shows the areas between the curves and the RMSEs and Table 3 shows their corresponding descriptive statistics.

The results show that the similarity between the UF and SUF flow rate curves increases with the increase in frame durations. This may be due to the flattening of sound artifacts when a larger number of samples is considered in one frame. The mean RMSE and area between the curves decrease from $2.81 \mathrm{ml} / \mathrm{s}$ and $27.67 \%$ for a frame duration of $0.2 \mathrm{~s}$ to $2.37 \mathrm{ml} / \mathrm{s}$ and $23.76 \%$ for a frame duration of $1 \mathrm{~s}$.

Two cases were found to be outliers with large non-overlaps between the UF and SUF curves. The two outlier cases are shown in Fig. 4 and offer insights into two main technical challenges that could be encountered with sonouroflowmetry.

The first issue is the potential presence of large sound artifacts as shown in Case (a). In Case (a), the SUF curve overshoots in the beginning possibly due to a manual urethral occlusion. Moreover, this subject voided a large volume causing the distance between the meatus and the water to majorly decrease during the voiding. This could have resulted in a relatively higher sound amplitude in the beginning of the recording. The second issue, shown 
(a)

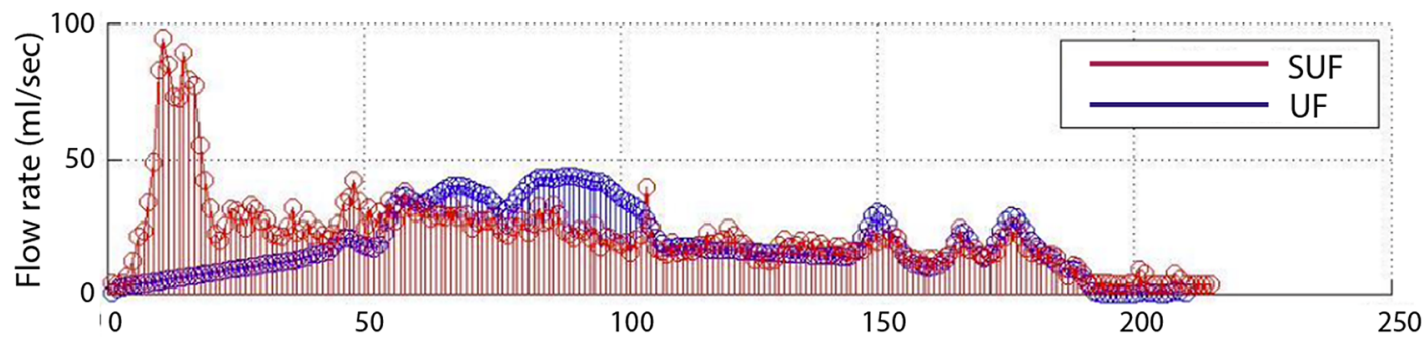

(b)

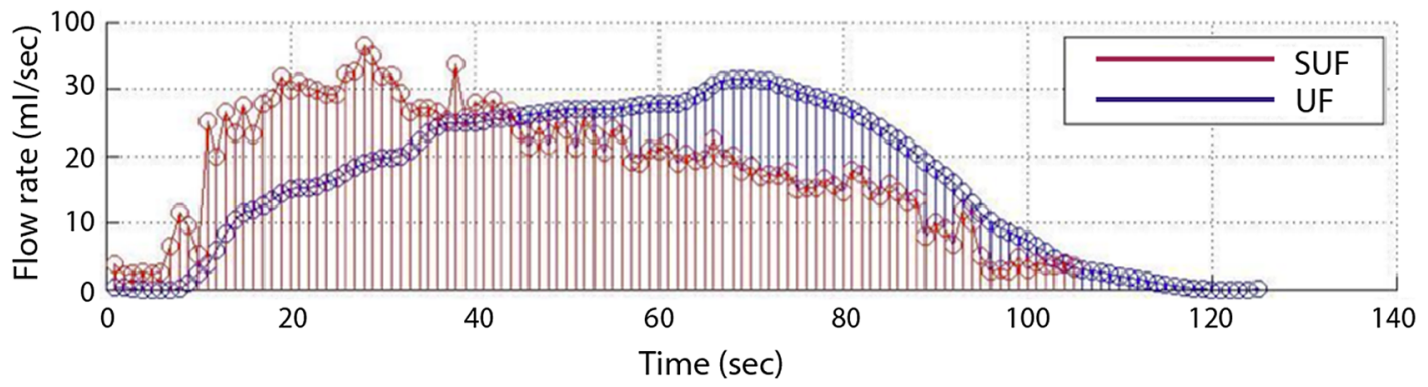

Figure 4. Cases with large non-overlaps between the UF and SUF flow rate curves. UF uroflowmetry, SUF sonouroflowmetry.
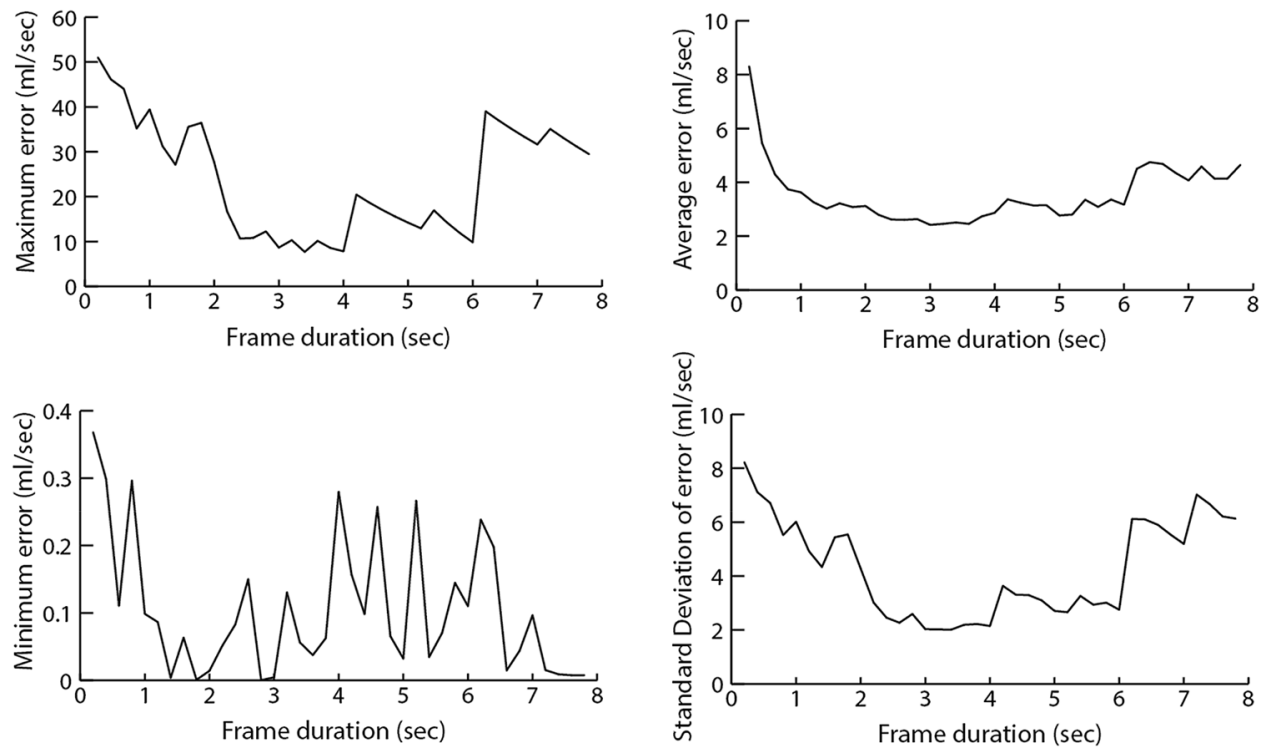

Figure 5. How $\mathrm{Q}_{\max }$ error rate statistics vary with frame durations.

in Case (b), is the overestimation of the flow rate at the beginning, which translates into an underestimation at a later time. This may be due to the low amount of water in the cup and its subsequent increase.

Accuracy of $\mathbf{Q}_{\max }$ estimation. To assess the accuracy of the proposed method in estimating $\mathrm{Q}_{\max }$, we report the errors associated with the estimations. Errors of estimation are calculated as the absolute values of the differences between UF and SUF based $\mathrm{Q}_{\max }$. The average, standard deviation, minimum and maximum errors are calculated over the whole dataset. Figure 5 shows how error rates vary with frame duration $f_{d}$.

The results show that the error rates are high for small frame durations, decrease with the increase of frame duration, reach a minimum, and then increase again. Frame durations between 2.5 and $3.5 \mathrm{~s}$ provide the lowest maximum, minimum, and average error rates, in addition to the lowest amount of variation in error.

Table 4 shows the statistics of error rates for select frame durations. Figure 6 shows UF versus SUF based $Q_{\max }$ measures for similar frame durations. 


\begin{tabular}{|l|l|l|l|l|}
\hline Frame duration & Minimum error & Maximum error & Mean error & SD of error \\
\hline $0.2 \mathrm{~s}$ & 0.37 & 51.02 & 8.30 & 8.22 \\
\hline $0.4 \mathrm{~s}$ & 0.30 & 46.15 & 5.45 & 7.11 \\
\hline $0.6 \mathrm{~s}$ & 0.11 & 44.03 & 4.29 & 6.71 \\
\hline $1 \mathrm{~s}$ & 0.10 & 39.42 & 3.63 & 6.01 \\
\hline $2.5 \mathrm{~s}$ & 0.06 & 9.26 & 2.50 & 2.12 \\
\hline $3.5 \mathrm{~s}$ & 0.08 & 7.23 & 2.41 & 2.01 \\
\hline $4 \mathrm{~s}$ & 0.28 & 7.83 & 2.87 & 2.15 \\
\hline $6 \mathrm{~s}$ & 0.11 & 9.84 & 3.17 & 2.75 \\
\hline
\end{tabular}

Table 4. $\mathrm{Q}_{\max }$ error rate statistics for different frame durations. SD Standard deviation.

\section{Discussion}

This study proposed a reproducible mobile sonouroflowmetry method that allows the estimation of the flow rate over time. As inputs, the method requires the measure of the total voided volume and a mobile phone recording of the sound of the urine stream. By linearly mapping the total sound energy to the total voided volume, the sound energy curve was transformed to a flow rate curve, allowing the estimation of the flow rate over time.

The accuracy of the method was evaluated using data collected from 44 young healthy men. $80 \%$ ( 44 out of 55 ) of the collected measurements we considered in the evaluation due to the presence of interfering sounds in $20 \%$ ( 11 out of 55 ) of the recordings. The results showed a strong correlation and high similarity between the SUF and the UF flow rates with a mean RMSE of $2.37 \mathrm{ml} / \mathrm{s}$ for a frame duration of $1 \mathrm{~s}$ and a high accuracy in estimating $\mathrm{Q}_{\max }$ with an average error of $2.41 \mathrm{ml} / \mathrm{s}$ for a frame duration of $3.5 \mathrm{~s}$.

The average normalized area between the UF and SUF curves was over $20 \%$. This could be attributed to (i) misalignments between the two curves caused by the manual cropping of the sound recordings and/or (ii) the rise of the water level inside the cup during the voiding which results in relatively higher energy levels in the beginning of the voiding. As the area under the curve is normalized using the total volume, an overestimation of flow rate in the beginning translates into an underestimation later on in time, which increases the area between the curves.

The study highlights the effect of the frame duration on the method's performance. The use of shorter frames led to an overestimation of the $\mathrm{Q}_{\max }$ and a higher RMSE. This is attributed to the impact of brisk sound artifacts on the energy estimation in small frames. The effect of these artifacts is diminished through the use of larger frames. However, when using large frames, we discarded the samples at the end of the sound signal that do not form a complete frame. This resulted in an overestimation of $\mathrm{Q}_{\max }$ as the total volume was being fitted into a duration shorter than the real voiding duration. In our study, these two effects led to an overestimation of the $\mathrm{Q}_{\max }$ in the majority of the recordings when using small and large frames. Future studies can examine ways to reduce the estimation errors attributed to sound artifacts and frame durations. A future direction to explore is the use of overlapping sliding frames which would allow the use of long frame durations while retaining the resolution of the flow rate curve. Sliding frames can also be useful for precisely detecting the start/end of voiding by minimizing the size of the segments to be discarded. In these cases, the optimal amount of overlap between the frames can be examined.

In addition to frame duration, the high-pass filter cutoff frequency $f_{c}$ and the energy calculation method may affect the performance. The proposed method uses a cutoff frequency of $1.2 \mathrm{kHz}$ and estimates the sound energy as the sum of the absolute values of the samples in a frame, rather than the sum of the squared values. These choices provided the best results following a series of trials, yet they may not be optimal. Further research is needed to inform the choice of cutoff frequencies and energy estimation methods for sonouroflowmetry applications.

Previous studies on sonouroflowmetry proved the feasibility of using mobile phone recordings to estimate flow parameters by reporting strong correlations between sound parameters and flow parameters ${ }^{10,24}$. However, only one previous study by Lee et al. aimed to estimate flow parameters by using a sound recording of a urine stream $^{26}$. Therefore, we will mainly compare our study with the study of Lee et al. ${ }^{26}$. The method proposed in this study uses the sound recording of the urine stream in conjunction with the total voided volume in order to estimate the flow parameters. The awareness of the total voided volume was essential to our method as it allowed the transformation of the sound energy curve into a flow curve. Lee et al.s method depended only on the sound recording of the urine stream without taking into consideration the total voided volume. However, the mapping of the sound features to flow parameters and the methods used to create the estimation models were not described. In other respects, the use of correlations and mean comparisons to assess the accuracy of the estimation model ${ }^{26}$ implies associations between truths and predictions, but does not provide an accuracy assessment of the models. As shown in our results, the accuracy of a sonouroflowmetry can be reported in terms of error rates that reflect the distance between real flow values and estimated flow values. Another evaluation aspect to consider is the similarity between flow curve patterns. As shown in our evaluation, the similarity between flow curves can be statistically assessed using a mixed-effects model approach and curve similarity measures. Visual comparisons are also necessary to assess whether the method's results would be clinically interpreted by physicians similarly to the results of a traditional uroflowmetry. However, such comparison necessitates multiple experts and a randomized experiment using a heterogeneous dataset of healthy subjects and subjects with LUTD. 


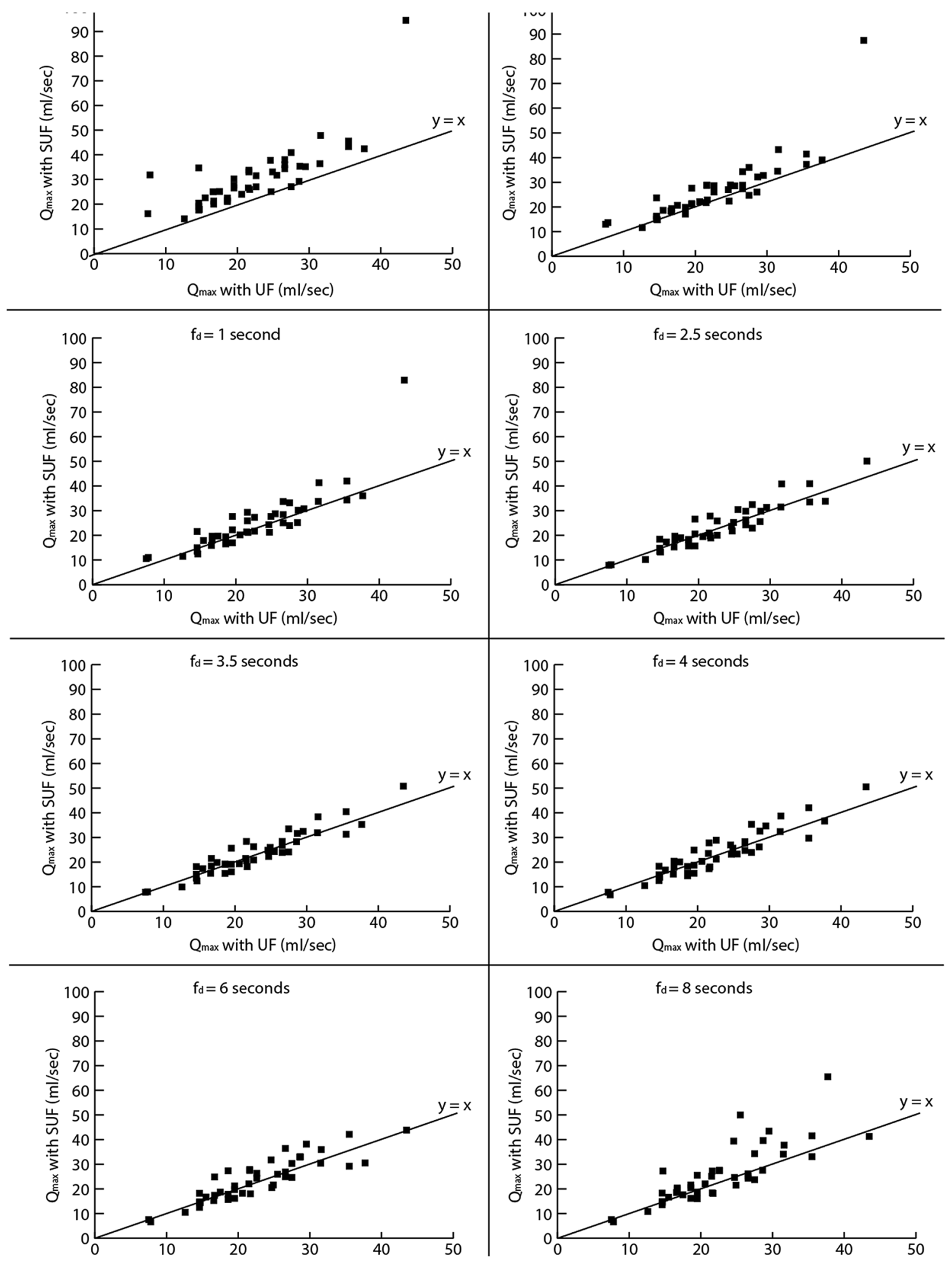

Figure 6. UF versus $S U F Q_{\max }$ for different frame durations.

This study has some limitations. First, the total voided volume was not automatically estimated; the patient was required to measure it themselves while collecting their urine. We consider this limitation acceptable since it is a simple process and is already a common practice for patients filling a bladder diary. Moreover, portable measuring cups were shown to be practical and to provide accurate measures of voided volume ${ }^{30}$. Second, the proposed method does not automatically detect the start and the end of the flow. Therefore, $\mathrm{Q}_{\text {avg }}$ cannot be automatically estimated. In order to improve the usability of sonouroflowmetry methods, our future work will examine ways to automate the detection of the beginning and end of the flow and the estimation of voided volume. These methods will have to rely on features other than the sound energy such as spectral features because 
using sound energy does not allow us to distinguish between micturition and other activities that can produce sound such as talking, breathing, knocking objects, or undressing.

Another limitation of our study is the removal of the funnel normally used when conducting uroflowmetries with weight-based uroflowmeters. The funnel reduces the kinetic energy of the fluid and is needed to decrease the noise in the weight signal. Without the funnel, weight-based uroflowmeters will tend to overestimate the flow rate across time. This added error is difficult to assess because it depends on the velocity of the urine hitting the surface, which is related not only to the flow rate, but also to the diameter of the urethra. Since the removal of the funnel is necessary for SUF, this represents an inevitable limitation when comparing SUF with weight based UF.

In terms of applicability, our study did not examine the accuracy of SUF with low flow rates. However, some of the cases that were part of our experiment included low flow rates and intermittent flows. These flow rates and intermittencies were visible in the SUF curves as shown in Fig. S8 in the Online Appendix. Therefore, we assume that the proposed method would also perform well in those cases. In other respects, previous studies noted that different methods may be needed to estimate flow parameters for males and females ${ }^{10,25,26}$. Our study considered young men with no LUTD urinating in a standing position. Future work needs to consider women and people with LUTD urinating both in standing and sitting positions. Additionally, multiple factors may affect the amplitude of sound recordings such as the sound conditions in the room where the measure takes place, the type of mobile device used to perform the recording, the height of the patient, the size and shape of the toilet bowl, the amount of water inside, and the distance or position of the recording device, among others ${ }^{22}$. Future studies can examine the accuracy of sonouroflowmetry methods in relation to these various factors.

As for the implications of this work, uroflowmetry is currently restrained by two well-known limitations: (1) the high intra-subject variability of flow parameters ${ }^{8,9}$, and (2) the inability to reproduce the natural process of voiding, since the patient should urinate on demand. To address these two limitations, the only possible solution is to conduct multiple uroflowmetries for each patient, all when the patient is physiologically ready ${ }^{8}$. Home uroflowmetry methods would make this possible; however, there are currently no cheap, practical, and accurate home-based methods that estimate the flow rate over time ${ }^{15,16}$. In this case, a reliable mobile sonouroflowmetry application could fulfill this gap as it enables the patients to conduct multiple flow analyses, while at home, and for a relatively low cost. If applied to patients with LUTD, this would enable continuous home-monitoring while providing objective patient-generated data, a valuable addition to the commonly-requested frequency volume charts and bladder diaries ${ }^{31,32}$. Such application would also help promote telemedicine by limiting office visits, while providing objective parameters to the clinician, especially in critical situations such as the recent COVID19 pandemic.

To conclude, we presented a new mobile sonouroflowmetry method that exploits the knowledge of the total voided volume to provide accurate estimations of the flow rate over time. The presented method estimates flow parameters from a sound recording of a urine stream hitting water using a frame-based energy calculation approach. Through a systematic evaluation approach, we showed that the method achieved a high level of accuracy in estimating the flow rate curve and the $\mathrm{Q}_{\max }$. There are significant potential gains offered by mobile sonouroflowmetry, yet further work is needed to establish it as a replacement, or a complement, to clinic-based uroflowmetry. Future studies on sonouroflowmetry can use the results of this work as a benchmark and aim to provide reproducible improvements.

\section{Data availability}

The data that support the findings of this study are available from the authors upon reasonable request and with permission of Saint Joseph University, Beirut, Lebanon.

Received: 25 February 2021; Accepted: 17 May 2021

Published online: 27 May 2021

\section{References}

1. Gravas, S. et al. Management of non-neurogenic male lower urinary tract symptoms (LUTS), incl. benign prostatic obstruction (BPO). (2019).

2. Rosier, P. Contemporary diagnosis of lower urinary tract dysfunction. F1000Research 8, 644 (2019).

3. Drake, M. J., Doumouchtsis, S. K., Hashim, H. \& Gammie, A. Fundamentals of urodynamic practice, based on International Continence Society good urodynamic practices recommendations. Neurourol. Urodyn. 37, S50-S60 (2018).

4. Siroky, M. B., Olsson, C. A. \& Krane, R. J. The flow rate nomogram: II. Clinical correlation. J. Urol. 123, 208-210 (1980).

5. Gratzke, C. et al. EAU guidelines on the assessment of non-neurogenic male lower urinary tract symptoms including benign prostatic obstruction. Eur. Urol. 67, 1099-1109 (2015).

6. El Helou, E. et al. Urodynamics in patients with multiple sclerosis: Is it necessary? A randomized-controlled trial. Scand. J. Urol. 55, 1-11 (2021).

7. Pessoa, R. \& Kim, F. J. Chapter 103-Urodynamics and voiding dysfunction. In Abernathy's Surgical Secrets 7th edn (eds Harken, A. H. \& Moore, E. E.) 452-454 (Elsevier, 2018).

8. Sonke, G. S. et al. A method for estimating within-patient variability in maximal urinary flow rate adjusted for voided volume. Urology 59, 368-372 (2002).

9. Feneley, M. R., Dunsmuir, W. D., Pearce, J. \& Kirby, R. S. Reproducibility of uroflow measurement: Experience during a doubleblind, placebo-controlled study of doxazosin in benign prostatic hyperplasia. Urology 47, 658-663 (1996).

10. Krhut, J. et al. Comparison between uroflowmetry and sonouroflowmetry in recording of urinary flow in healthy men. Int. J. Urol. 22, 761-765 (2015).

11. Kranse, R. \& van Mastrigt, R. Causes for variability in repeated pressure-flow measurements. Urology 61, 930-934 (2003).

12. Dabhoiwala, N., Osawa, D., Lim, A. T. L. \& Abrams, P. The ICS-'BPH' study: Uroflowmetry, lower urinary tract symptoms and bladder outlet obstruction. Br. J. Urol. 82, 619-623 (1998).

13. Gammie, A., Rosier, P., Li, R. \& Harding, C. How can we maximize the diagnostic utility of uroflow?: ICI-RS 2017. Neurourol. Urodyn. 37, S20-S24 (2018). 
14. Sonke, G. S. et al. Low reproducibility of maximum urinary flow rate determined by portable flowmetry. Neurourol. Urodyn. 18, 183-191 (1999).

15. Bray, A., Griffiths, C., Drinnan, M. \& Pickard, R. Methods and value of home uroflowmetry in the assessment of men with lower urinary tract symptoms: A literature review. Neurourol. Urodyn. 31, 7-12 (2012).

16. Chun, K., Kim, S. J. \& Cho, S. T. Noninvasive medical tools for evaluating voiding pattern in real life. Int. Neurourol. J. 21, S10 (2017).

17. Alothmany, N. et al. Critical review of uroflowmetry methods. J. Med. Biol. Eng. 38, 685-696 (2018).

18. Park, S. et al. A mountable toilet system for personalized health monitoring via the analysis of excreta. Nat. Biomed. Eng. 4, 624-635 (2020).

19. Nesbit, R. M. \& Baum, W. C. Obstructive uropathy in childhood; diagnosis and surgical management. J.-Michigan State Med. Soc. 54, 1067 (1955).

20. Keitzer, W. A. \& Huffman, G. C. The voiding audiograph: A new voiding test. J. Urol. 96, 404-410 (1966).

21. Koiso, K., Nemoto, R. \& Ohtani, M. Urophonographic studies of benign prostatic hypertrophy. J. Urol. 145, 1071-1077 (1991).

22. Hurtík, P., Burda, M., Krhut, J., Zvara, P. \& Lunácek, L. Automatic diagnosis of voiding dysfunction from sound signal. In 2015 IEEE Symposium Series on Computational Intelligence 1331-1336 (2015). https://doi.org/10.1109/SSCI.2015.190.

23. Hitt, D., Zvarova, K. \& Zvara, P. Urinary flow measurements via acoustic signatures with application to telemedicine. In 39th AIAA Fluid Dynamics Conference 4300 (2009).

24. Zvarova, K. et al. Recording urinary flow and lower urinary tract symptoms using sonouroflowmetry. Can. J. Urol. 18, 5689 (2011).

25. Gärtner, M. et al. Evaluation of voiding parameters in healthy women using sound analysis. Lower Urin. Tract Symp. 10, 12-16 (2018).

26. Lee, Y. J., Kim, M. M., Song, S. H. \& Lee, S. A novel mobile acoustic uroflowmetry: Comparison with contemporary uroflowmetry. J. Korean Continence Soc. https://doi.org/10.5213/inj.2040250.125 (2021).

27. Gammie, A. et al. International Continence Society guidelines on urodynamic equipment performance. Neurourol. Urodyn. 33, 370-379 (2014).

28. Van der Elst, W., Molenberghs, G., Hilgers, R.-D., Verbeke, G. \& Heussen, N. Estimating the reliability of repeatedly measured endpoints based on linear mixed-effects models. A tutorial. Pharmaceut. Stat. 15, 486-493 (2016).

29. Shan, G., Zhang, H. \& Jiang, T. Correlation coefficients for a study with repeated measures. Comput. Math. Methods Med. 2020, e7398324 (2020).

30. Yamada, M., Tanaka, K., Yamaguchi, O. \& Kawabe, K. A new portable measuring cup for voided volume. Int. J. Urol. 8, 350-352 (2001).

31. Rosier, P. F. et al. International continence society good urodynamic practices and terms 2016: Urodynamics, uroflowmetry, cystometry, and pressure-flow study. Neurourol. Urodyn. 36, 1243-1260 (2017).

32. Rubilotta, E. et al. Is the voided volume at office uroflowmetry physiological and reliable? A comparison between voiding diary and uroflowmetry. Urol. Int. 104, 908-913 (2020).

\section{Acknowledgements}

This research was supported by the Institutional Research Committee of Saint Joseph University, Beirut, Lebanon and by JSPS Kakenhi, Grant Number JP20K20244.

\section{Author contributions}

E.E.H. and K.Y. conceived the method(s), E.E.H., J.N. and K.Y. conducted the experiment(s), E.E.H., K.Y., G.S. and S.H. conducted the evaluation(s), E.EH., J.N., K.Y., G.M., G.S. and S.H. analysed the results, and wrote and reviewed the manuscript.

\section{Competing interests}

The authors declare no competing interests.

\section{Additional information}

Supplementary Information The online version contains supplementary material available at https://doi.org/ 10.1038/s41598-021-90659-9.

Correspondence and requests for materials should be addressed to E.E.H. or S.H.

Reprints and permissions information is available at www.nature.com/reprints.

Publisher's note Springer Nature remains neutral with regard to jurisdictional claims in published maps and institutional affiliations.

(c) (i) Open Access This article is licensed under a Creative Commons Attribution 4.0 International License, which permits use, sharing, adaptation, distribution and reproduction in any medium or format, as long as you give appropriate credit to the original author(s) and the source, provide a link to the Creative Commons licence, and indicate if changes were made. The images or other third party material in this article are included in the article's Creative Commons licence, unless indicated otherwise in a credit line to the material. If material is not included in the article's Creative Commons licence and your intended use is not permitted by statutory regulation or exceeds the permitted use, you will need to obtain permission directly from the copyright holder. To view a copy of this licence, visit http://creativecommons.org/licenses/by/4.0/.

(c) The Author(s) 2021 